\section{Implicancias culturales en la morfología craneana de grupos étnicos pre-colombinos peruanos}

\author{
Cultural implications in the cranial morphology of peruvian pre- \\ columbian ethnic groups
}

\begin{abstract}
Resumen
El estudio consiste en relacionar el agente causal de las deformaciones cefálicas con el área cultural, el cierre sutural y las formas craneanas en el contexto del crecimiento craneofacial del poblador peruano precolombino, comprendidos en la clasificación tipológica de Weiss. Muestra: dieciocho cráneos representativos de grupos étnicos precolombinos del antiguo Perú comprendido dentro de la clasificación tipológica de Weiss, seleccionados de la curaduría de restos humanos del Museo Nacional de Arqueología, Antropología e Historia del Perú. Metodología: Se procedió con el reconocimiento de los cráneos y el registro de los códigos respectivos adoptada por la curaduría según las asociaciones culturales, regionales determinadas. Luego los cráneos fueron fotografiados en todas sus normas anatómicas. La información obtenida fue a través de la observación de imágenes de alta resolución. Resultados y conclusiones: Las deformaciones cefálicas son congruentes con la ley de Virchow. En el Perú precolombino se resume dos filums, el Llautu asociado a culturas andinas y la Cuna a pueblos costeños. El Llautu es concordante con el cierre precoz de la sutura sagital y en consecuencia el cerebro como materia orgánica influye en el bregma y la sutura coronal estimulando la osificación de un cráneo dolicocéfalo de aspecto anular. La cuna es concordante con el cierre precoz de la sutura coronal y en consecuencia el cerebro en crecimiento estimula las membranas suturales: sagital, parietoesfenoidal y parietotemporal orientando la osificación de un cráneo braquicéfalo de aspecto tabular.
\end{abstract}

Palabras Claves: Deformaciones craneanas, antropología cultural peruana.
Artículo Original
Luis Hernando Gálvez Calla'; Luis Maita Velizz; †Celso Guillén Borda ${ }^{3} ;$ Leoncio Menéndez Méndez ${ }^{3}$
1. Departamento Académico de Ciencias Básicas. 2. Departamento Académico Médico Quirúrgico.

3. Departamento Académico de Estomatología Pediátrica.

1-3 Facultad de Odontología, Universidad Nacional Mayor de San Marcos, Perú.

\section{Correspondencia:}

Dr. Luis H. Gálvez Calla

Facultad de Odontología, Universidad Nacional Mayor de San Marcos, Av. German Amézaga s/n, Lima 1, Perú.

Correo electrónico: lugalca2005@gmail.com

\begin{abstract}
The objective of the study was to relate the causative agent of cranial deformations with the cultural area, the suture closure and cranial forms, in the context of the craniofacial growth of pre-columbian peruvian population, included in the typological classification of Weiss. Sample: eighteen skulls representing pre-columbian ethnic groups of ancient Peru comprised within the typological classification of Weiss were selected from the curatorship human remains of National Museum of Archaeology, Anthropology and History of Peru. Methodology: first we made the recognition of the skulls and registered their codes adopted according to regions or cultural associations by Curatorship. Then the skulls were photographed following anatomical standards. The information obtained was through observation of high-resolution images. Results and conclusions: cephalic deformations are consistent with the law of Virchow. Pre-columbian Peru summarizes two phylum, "llautu" associated with andean cultures and "cuna" to coastal towns. Llautu is consistent with the early closure of the sagittal suture and consequently the brain as organic matter influences the bregma and the coronal suture stimulating an ossification of a dolichocephalous cranium of annular aspect. "Cuna" is concordant with the early closure of the coronal suture and consequently the brain in growth stimulates suture membranes: sagittal, parietosphenoidal and parietotemporal, leading to brachycephalic skull ossification of a tabular appearance
\end{abstract}

Keywords: Cranial deformation, peruvian cultural anthropology.

\section{Introducción}

La existencia de costumbres prehispánicas en ciertos pueblos sudamericanos de practicar deformaciones cefálicas en niños, han sido reportados por primera vez por Don Cieza De León en sus Crónicas del Perú-1553 sin embargo, las modificaciones corporales con propósitos culturales o sociales como las deformaciones cefálicas datan desde tiempos inmemoriales ${ }^{2}$, que han sido practicadas por diversos grupos étnicos en casi todas las civilizaciones, culturas o regiones, alcanzando la mayor frecuencia y variación en las culturas Maya, Inca, Tiwanaku y diversas otras culturas andinas ${ }^{3,4,5}$.

La deformación cefálica intencional, por sí sola no significó mucho salvo cuando está asociado culturalmente con otros condiciones sociales mágico-religiosas ${ }^{6,7,8}$, estéticos ${ }^{9}$, cosméti$\cos ^{10}$ y de estatus de guerra ${ }^{11}$; siendo
Fecha de recepción: 31-01-14 Fecha de aceptación: 19-06-14

erróneo estudiar al hombre y a las civilizaciones antiguas solo desde la perspectiva social y económica, olvidando el aspecto mágico y religioso que en esa época era trascendente en cualquier actividad del hombre ${ }^{12}$.

La diversidad de deformaciones craneanas artificiales precolombinas estaba asociada a un tipo específico de instrumento deformador ${ }^{13,14,15}$; aplicado al niño por su propia madre desde el momento de nacer (fig. $1, \mathrm{E})^{16}$ 
Desde el punto de vista del tipo de la deformación craneana intencional como producto de un aparato deformador bien definido, se distinguen tres tipos de deformación. Un tipo anular producido por vendas y dos tipos tabulares producidos por tablillas libres y por medio de cunas ${ }^{13,15}$; existiendo una alta densidad de anulares en el norte de Chile, sur del Perú y altiplano Perúboliviano ${ }^{8}$; las categorías más comunes de deformaciones craneanas en los Andes son las de tipo circular y tabular ${ }^{17}$. Muchas poblaciones del norte de Chile fueron influenciadas durante siglos por la cultura Tiawanaku, que predominaba la deformación craneana circular característica de los tiawanacotas ${ }^{18}$

Las formas tabulares resultan de la compresión de las partes anterior y posterior de la caja craneana por medio de almohadillas o tablas rígidas, creando una expansión pronunciada del ancho craneal; mientras que las formas circulares eran producidas por la aplicación de vendas alrededor del cráneo, con la consiguiente reducción en el ancho craneal y un aumento compensatorio en su longitud ${ }^{2}$, que modelan cráneos braquicefálicos y dolicocefálicos según García Hernández \& Murphy Echevarría $^{18 .}$

Pedro Weiss ${ }^{19,20}$ logró desarrollar una clasificación sobre bases estrictamente morfológicas y definió tipos que eran característicos para algunos períodos culturales del Perú. Aunque destaca el valor local de ellos, demuestra su presencia en otros lugares de América.

En el antiguo Perú las deformaciones cefálicas encuentran sus variantes más extremas agrupándose en dos grandes filums, el costeño o de cuna y el serrano o de llautus.

La deformación craneana artificial tipo Natchez descrita por Weiss ${ }^{19}$, en alusión a la forma craneal de los indígenas Natchez de la cuenca inferior del Mississippi, definida por Imbelloni ${ }^{19,21}$ como tipo "tabular erecto de grado extremo", conocida en la literatura antropológica universal como increíbles por el extremo de su distorsión; los Natchez peruanos del estudio Osteo cultural de Weiss $^{19}$ es una expresión posiblemente social, pero no cultural; resultan forjados por llautu con rosca paracas o almohadilla de algodón en la parte posterior. Hay formas Natchez que no llegan extremos de distorsión que merezcan el calificativo de increíbles.

La construcción y el uso de artefactos deformadores requerían al menos de un cierto grado de conocimientos físicos y biológicos, siendo necesarios el conocimiento de las estructuras craneofaciales del infante y el niño, consolidación de fontanelas y cierre cronológico de suturas $^{22}$ con la finalidad de interactuar con los procesos de osificación normal (Tabla 1).

La bóveda craneana al nacimiento es asimétrica por la presión a la que está sometida por efectos del parto; el cual se corrige con el crecimiento post na$\mathrm{tal}^{23}$.

Estudios anátomo-funcionales, ontogenéticos (evolución individual) y filogenéticos (evolución como especie), indican que, "el crecimiento de la bóveda craneana está ligado al crecimiento del cerebro, que influye además a la base endocondral ${ }^{24,25,26}$. Son un claro ejemplo la anancefalia y la hidrocefalia.

Los campos de crecimiento que gobiernan y controlan el crecimiento óseo se encuentran en los tejidos blandos circundantes al hueso ${ }^{27}$; al igual que el impulso genético y las células que participan en el proceso de crecimiento craneofacial ${ }^{27}$; denominado matriz funcional por Melvin Moss ${ }^{27}$.

Se ha demostrado, que el desmocraneo cambia mucho más que el condrocraneo durante el crecimiento normal ${ }^{28}$, se encuentra primordialmente bajo la influencia de factores epigenéticos generales y ambientales generales ${ }^{23,29,30,31}$.

La presión que ejerce el cerebro en crecimiento expande las suturas del desmocráneo y en consecuencia el crecimiento de la bóveda craneal. Según Moss $^{27}$ el hueso no es un determinante de su propio crecimiento, los factores de crecimiento se encuentran en los tejidos asociados (matriz funcional)

El crecimiento sutural y periostico son gobernados adicionalmente por influencias ambientales locales ${ }^{30,31}$; sin embargo para Proffit ${ }^{32}$, las suturas y tejido perióstico no son los determinantes fundamentales del crecimiento cráneofacial, afirmando que las suturas carecen de potencial de crecimiento innato y que el crecimiento a nivel de suturas responden a influencias externas. Concluyendo que las suturas son lugares de crecimiento pero no centros de crecimiento.

Retzlaff ${ }^{33}$ confirmó que no había ningún indicio de osificación completa de las suturas y que depende de las fuerzas que inciden sobre ella.

Las fontanelas dan la flexibilidad necesaria para el movimiento de los huesos craneales durante el parto y el período de rápido crecimiento cerebral. Estas se reducen a dimensiones suturales después del nacimiento ${ }^{22}$ (Tabla 1)

La fusión sinostósica del cráneo empieza al cabo del $1^{\circ}$ año después del nacimiento con la sutura metópica que se consolida alrededor de los 7 años de edad, le siguen, en el orden siguiente: las suturas sagital, coronal y lamboi$\operatorname{dea}^{22,34}$, entre los 22 y 35 años (Tabla 2).

La valoración del grado de cierre sutural fue elaborado por Meindl y Levejoy ${ }^{35}$ modificado de la forma siguiente:

Cs = Cierre sutural, sin evidencia de osificación ectocraneal (seoe)

Sm = sinóstosis mínima, formación de un puente óseo mínima alrededor de la sutura que puede alcanzar hasta el 50\% de la sinóstosis del sitio

Ss = sinóstosis significativa. Grado marcado de obliteración, sin alcanzar el cierre completo

St $=$ sinostosis total, fusión completa del sitio

El cierre prematuro produce distorsiones craneales concordantes con la ley de Virchow $^{36}$.

Virchow en $1851^{36}$, acuño el término de craneosinostosis para describir la fusión prematura de las suturas craneanas y formuló la "ley de Virchow" que dice que la sinostosis inhibe el crecimiento normal del cráneo perpendicularmente a la sutura fusionada, mientras continua su crecimiento en el sentido paralelo $^{36}$ (Tabla 3).

El presente estudio pretende relacionar el agente causal de las deformaciones craneanas precolombinas del antiguo Perú con el área cultural de los grupos poblacionales, el cierre sutural y las formas craneanas del crecimiento craneofacial, congruentes con la ley de Virchow $^{36}$

\section{Materiales y Método}

El estudio consiste en relacionar el agente causal de las deformaciones cefálicas con el área cultural, el cierre sutural y las formas craneanas del crecimiento craneofacial del poblador precolombino del antiguo Perú. Se emplearon dieciocho cráneos representativos de grupos étnicos precolombinos del antiguo Perú comprendido dentro de la clasificación tipológica de Pedro Weiss ${ }^{19}$, preservados como patrimonio cultural en la curaduría de restos humanos del $\mathrm{Mu}$ seo Nacional de Arqueología, Antro- 
pología e Historia del Perú; por esta razón no recibieron ningún tipo de intervención estando solo autorizado para el análisis del cierre sutural según el método de valoración de Meindl y Lovejoy $^{35}$, y la observación macroscópica de las formas craneanas, por lo que la muestra fue íntegramente fotografiada en toda sus normas anatómicas; tomadas con cámara digital Lumix Panasomic DMC-FZ35, 18x optical zoom, 12.1 megapixels, full HD. La información obtenida fue a través de la observación de imágenes de alta resolución.

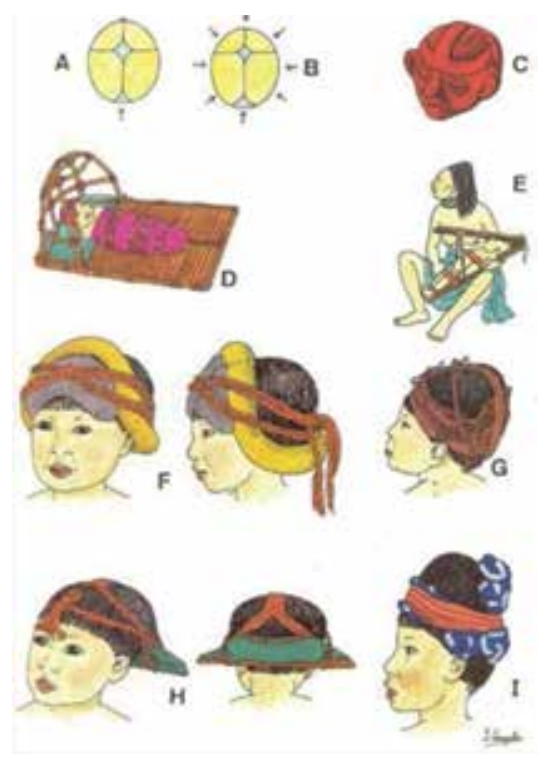

Se inició con el reconocimiento de los cráneos y el registro de los códigos respectivos adoptada por la curaduría según las asociaciones culturales y regionales de pertinencia a la clasificación tipológica de Weiss. Se procedió a describir morfológicamente cada uno de los cráneos deformados por todas sus normas y registrar las características del cierre sutural según el método de valoración del Meindl y Lovejoy. Los datos se procesaron en tablas de $4 \times 3$, 4x4 y una tabla resumen de $7 \times 4$ con la finalidad de establecer relación horizontal lógica entre las variables agente causal, área cultural, cierre sutural y forma craneal de los cráneos deformados comprendidos en la muestra.

\section{Resultados}

En la Tabla 4 se muestran los resultados. De los 18 cráneos examinados, 3 cráneos deformados por cuna presentaron deformación tabular por sinostosis total de la sutura coronal que corresponden a los tipos Fronto Occipital e Inca Costeño de Weiss, de forma craneal braquicefálica y área cultural costeño (Fig.2).

\section{Fig. 1: Artefactos y métodos de deformación craneal en culturas andinas}

(A) Comprensión en sentido entero-posterior;

(B) Comprensión circular;

(C) Figura de cerámica que mediante un aparejo;

(D) Cuna Diseñada para la comprensión craneal;

(E) Madre sosteniendo a su hijo de meses con un aparejo formando por dos tablas, para lograr una deformación anteroposterior;

(F) y $(G)$ Aparejos deformatorios formados por cuerdas, rollos de tela y placas de cuero;

(H) Aparejo formado por una placa de madera almohadillada y sujeta por cordajes;

(I) Deformación mediante la aplicación circular de vendas tomado de la obra de Domingo Campillo, Paleopatología, Fundación Uriach, 1994.

Publicada por Onnega en celtiverian.net

www.celtiveria.net/verrespuesta.asp? idp $=10179$

Tabla 1: Cierre de fontanelas*

\begin{tabular}{|l|c|}
\hline Fontanelas & Osificación después de nacimiento \\
\hline Fontanela anterior & Durante el $2^{\circ}$ años \\
\hline Fontanela posterior & $2-3$ meses \\
\hline Fontanela antero-laterales (2) & 3 meses \\
\hline Fontanela postero-laterales (2) & Fines del $1^{\circ}$ años \\
\hline
\end{tabular}

*Información obtenida de Sperber GH. Craniofacial Embriology 4th. Edition. Wright 1989.

Tabla 2: Épocas de cierre de alguna suturas craneo faciales en humanos*

\begin{tabular}{|l|c|c|c|}
\hline \multicolumn{1}{|c|}{ Sutura craneal } & $\begin{array}{c}\text { Comienzo de } \\
\text { cierre (años) }\end{array}$ & Sutura facial & $\begin{array}{c}\text { Comienzo de } \\
\text { cierre (años) }\end{array}$ \\
\hline Interfrontal o metópica & 2 & Intermaxilar o palatina & 30 a 35 \\
\hline Interparietal o sagital & 22 & Frontomaxilar & 68 a 71 \\
\hline Frontoparietal o coronal & 24 & Frontonasal & 68 \\
\hline \multirow{2}{*}{ Occipitoparietal o lambdoidea } & 26 & Nasomaxilar & 68 \\
\cline { 2 - 4 } & & Frontocigomática & 72 \\
\hline Temporoparietal & $35-39$ & Cigomáticomaxilar & 70 a 72 \\
\hline
\end{tabular}

${ }^{*}$ Modificado de Meike MC. Craniofacial Development. Growth and Evolution. 1 st.edition. Bateson Publishing Bressingham. Norfolk. England. 2002 
Tabla 3: Formas de la cabeza y compromiso sutural

\begin{tabular}{|l|l|}
\hline Formas de la cabeza & Sutura fusionada o ausente \\
\hline Dolicocefalia o escafocefalia & Sagital \\
\hline Braquicefalia & Coronal bilateral \\
\hline Plagiocefalia anterior & Coronal unilateral \\
\hline Plagiocefalia posterior & Lambdoidea unilateral \\
\hline Trigonocefalia & Metópica \\
\hline
\end{tabular}

Modificado de Sperber GH. Craniofacial Embriology. 4 th edition. Wright 1989

Tabla 4: Agente causal de las deformaciones cefálicas y área cultural, cierre sutural y forma craneana del poblador peruano precolombino

\begin{tabular}{|c|l|l|l|l|l|l|}
\hline $\begin{array}{l}\text { No de } \\
\text { cráneos }\end{array}$ & $\begin{array}{l}\text { Cráneos deformados } \\
\text { precolombinos Tipolo- } \\
\text { gía de Weiss }\end{array}$ & $\begin{array}{l}\text { Agente causal (Artefacto } \\
\text { deformador) }\end{array}$ & Área cultural & Cierre sutural & $\begin{array}{l}\text { Forma craneana } \\
\text { (Visual) }\end{array}$ & $\begin{array}{l}\text { Deformación } \\
\text { cefálica de } \\
\text { Imbelloni }\end{array}$ \\
\hline 2 & $\begin{array}{l}\text { Fronto occipital } \\
\text { Inca costeño }\end{array}$ & Cuna & Costeño & Stse coronal & Braquicefalia & Tabular \\
\hline 2 & $\begin{array}{l}\text { Aimara } \\
\text { Opa }\end{array}$ & Llautu & Andino & Ss lambdoidea & Dolicocefalia & Anular \\
\hline 2 & $\begin{array}{l}\text { Cavernas } \\
\text { Huaura }\end{array}$ & Llautu & Andino & $\begin{array}{l}\text { Cs coronal seoe } \\
\text { Sm sagital }\end{array}$ & $\begin{array}{l}\text { Braquicefalia } \\
\text { Dolicocefalia }\end{array}$ & $\begin{array}{l}\text { Cuneiforme } \\
\text { Tabular }\end{array}$ \\
\hline 2 & Cabeza larga & $\begin{array}{l}\text { Llautu + rosca } \\
\text { Paracas }\end{array}$ & Andino & Ss coronal & Braquicefalia & $\begin{array}{l}\text { Anular } \\
\text { Tabular }\end{array}$ \\
\hline 2 & Necropolis & Paracas & Andino & Ssse coronal & Braquicefalia & Seudoanular \\
\hline 1 & Nazca & $\begin{array}{l}\text { Llautu + rosca } \\
\text { Paracas+almohadilla }\end{array}$ & Andino & Ss sagital & Dolicocefalia & $\begin{array}{l}\text { Cuneiforme } \\
\text { Trabular }\end{array}$ \\
\hline
\end{tabular}

$\mathbf{S m}=$ sinostosis mínima; $\mathbf{S} \boldsymbol{s}=$ sinostosis significativa, $\mathbf{S s s e}=$ sinestosis significativa supraestefanium; $\mathbf{S t}=$ sinostosis total; $\mathbf{S t s e}=$ sinostosis total supraestefanium; $\mathbf{C}$ = cierre de sutura; seoe $=$ sin evidencia de osificación ectocraneal.

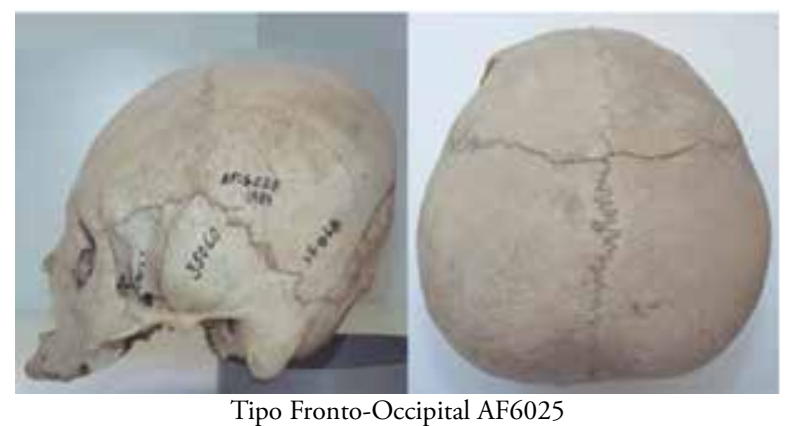

Fig.2: Deformación tabular oblicuo por cuna que modela cráneo braquicefálico de área cultural costeño

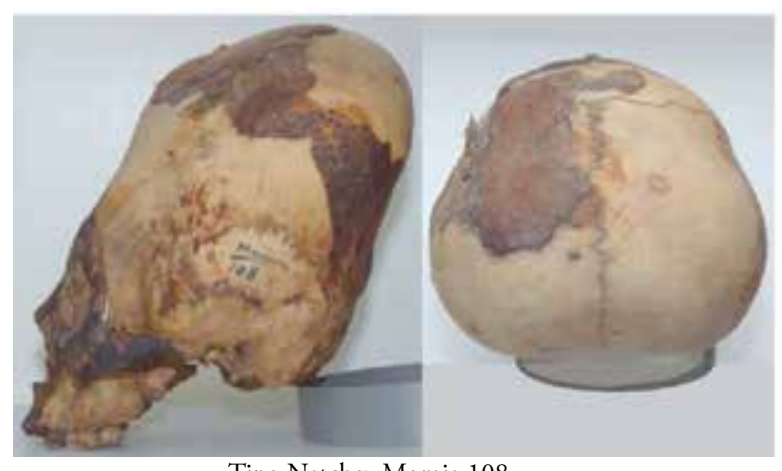

Tipo Natchez Momia 108

Fig.4: Deformación tabular erecto por llautu que modela cráneo braquicefalico de área cultural andino

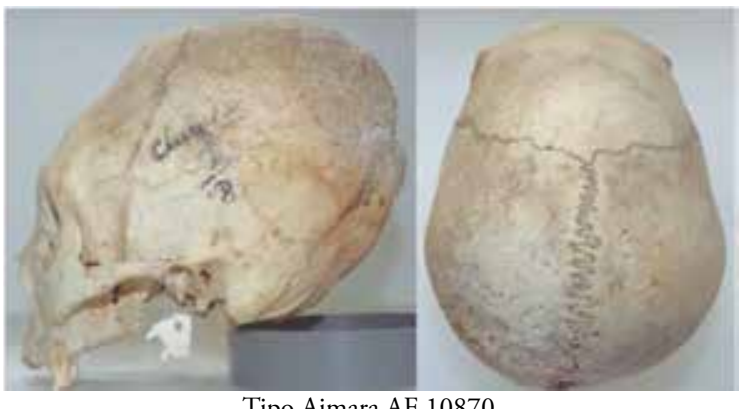

Tipo Aimara AF 10870

Fig. 3: Deformación anular por llautu que modela cráneo dolicocéfalo de área cultural andino

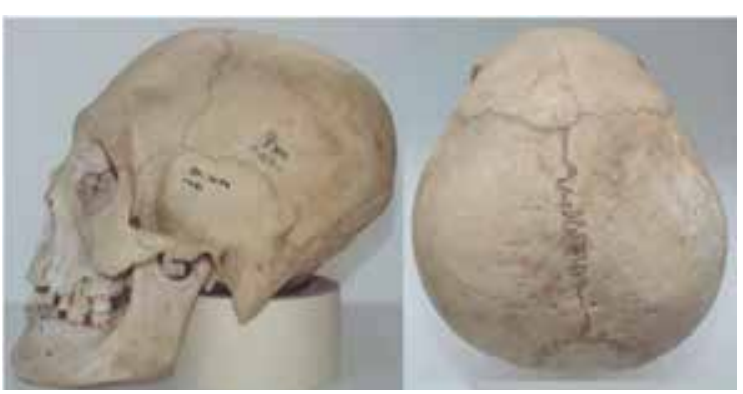

Tipo Huaura AF 1699

Fig.5: Deformación tabular oblicuo por llautu que modela cráneo dolicocefálico, de área cultural andino 
De los 18 cráneos examinados, 4 cráneos deformados por llautu presentaron deformación anular por sinostosis significativa de la sutura lambdoidea que corresponden a los tipos Aimara y Opa de Weiss, de forma craneal dolicocefálica de área cultural andino (Fig.3).

Las formas braquicefálicas, producto de la sinostosis coronal, de cráneos deformados por llautu y rosca de algodón paracas corresponden al tipo Cabeza Larga (2), Necrópolis (2) y Natchez (1) de Weiss, de área cultural andino (Fig.4). Las formas dolicocefálicas, por sinostosis total o significativa de la sutura sagital, de cráneos deformados por llautu y rosca de algodón paracas corresponden a los tipos Huaura (2), Natchez (1) y Nazca (1), de área cultural andino (Fig.5).

\section{Discusión}

Estudios ontogenéticos y filogenéti$\cos ^{24,25,26}$ indican que el crecimiento de la bóveda craneal está ligado al crecimiento del cerebro como materia orgánica, según matriz funcional de $\mathrm{Moss}^{27}$, que el crecimiento no está en el propio hueso sino que se debe al desarrollo sutural $^{27}$, gobernados adicionalmente según Limborgh ${ }^{30.31}$ por influencias ambientales. De manera que en el contexto de los procesos de crecimiento craneofacial de la población precolombina, comprendidos en la tipología de Weiss ${ }^{19}$, los artefactos deformadores tipos llautu y cuna, indentificados como factores ambientales (cultural) influyeron y contribuyerón categóricamente a la deformación craneana en los antiguos peruanos precolombinos y de otros lugares del continente americano.

La compresión fronto-occipital con artefactos rígidos utilizados en la deformación craneana de tipo tabular e identificados en el presente estudio, posiblemente aplicados antes que la bregmática se cierre produjo cambios en la dirección del crecimiento óseo por remodelación de los segmentos óseos del frontal que los aproxima a los parietales, reduciendo el espacio bregmático y el espacio de la sutura coronaria y en consecuencia se aceleran los mecanismos del cierre sutural respectivo. En estas condiciones el cerebro en crecimiento, como materia orgánica, orienta el crecimiento de la calota craneal hacia arriba y atrás en función de las suturas frontoesfenoidal, parietoesfenoidal, parietotemporal y parietoccipital; y transversalmente a través de la sutura sagital, modelando cráneos braquicefálicos. Estos resultados son congruentes con los estudios antropológicos de TorresRouff', Munizaga ${ }^{8}$, y Dembo \& Imbelloni ${ }^{17}$.

En las deformaciones craneanas de tipo anular por Llautu, el tejido o banda que rodea la cabeza del niño, atadas fuertemente por cordones o ligaduras, comprimen la sutura sagital y en consecuencia aceleran los mecanismos del cierre sutural respectivo; en tal sentido la masa orgánica del cerebro en crecimiento ejerce presión sobre las regiones bregmática y lambdoidea de la calota craneal ampliando sus espacios y estimulando su osificación intramenbranosa; orientando el crecimiento anteroposterior dolicocefálico del neurocraneo. Los resultados de este estudio son congruentes con los estudios antropológicos realizados por Torres-Rouff ${ }^{2}$, Munizaga ${ }^{8}$, y Dembo \& Imbelloni ${ }^{17}$

\section{Conclusiones}

1.- El presente estudio colige que hay relación directa entre el agente causal y el área cultural, el cierre sutural y las formas craneanas en el contexto del crecimiento cráneofacial, congruente con la ley de Virchow: "El cráneo crece en el sentido de la sutura que se cierra precozmente".

2.- Son muchos los artefactos empleados en las deformaciones cefálicas. En el antiguo Perú precolombino se resume la existencia de dos filums, deformación por el Llautu asociado a culturas andinas y deformación por Cuna asociado a pueblos costeńos.

3.- El Llautu que rodea la cabeza del nińo cierra precozmente la sutura sagital $y$ en consecuencia el cerebro en crecimiento, como materia orgánica, ejerce presión a la región frontal tensionando las membranas del bregma y la sutura coronal estimulando la osificación de un cráneo dolicocefálico de aspecto anular.

4.- La cuna y/o tablillas rígidas o semirrígidas aplicadas a la cabeza del niño, cierra precozmente la sutura coronal $y$ en consecuencia el cerebro en crecimiento, como materia orgánica, ejerce presión a las regiones parietales tensionando las membranas periósticas de la sutura sagital, parietoesfenoidal y parietotemporal estimulando la osificación de un cráneo braquicefálico de aspecto tabular.

\section{Agradecimiento}

Nuestro sincero agradecimiento al Museo Nacional de Arqueología, Antropología e Historia del Perú, por las facilidades técnicas y administrativas que hicieron posible el logro de los objetivos del presente estudio.

\section{Referencias bibliográficas}

1. Adrada J. La deformación craneana entre los pueblos primitivos de Sudamérica. Estudios geográficos. org. [Online] consultado 2013 Marzo 25; [7]. Disponible en: URLhttp://www.estudiosgeograficos.org/Reportajes/Deformacion\%20Craneana.pdf

2. Torres-Rouff C. La deformación craneana en San Pedro de Atacama. Estudios Atacameños: Arqueología y Antropología Surandina. 2007;33:25-38.

3. Boada RA. La deformación craneana como marcador de diferenciación social. Museo del Oro, Banco de la República, Boletín del Museo de Oro.1995 Ene-Dic; $\mathrm{N}^{\circ}$ 38-39:135-147.

4. Torres-Rouff C. y Yablonsky L. Cranial vault modification as a cultural artifact: A comparison of the Eurasian steppes and the Andes. Homo. Journal of Comparative Human Biology. 2005;56(1):1-16.

5. Borja VC, Gálvez CL. Deformaciones cefálicas en el antiguo Perú. Odontol Sanmarquina 2006;9(1):31-35.

6. López RE. La deformación intencional cefálica en los antiguos habitantes de departamento de Oruro. La Paz: Universidad Técnica de Oruro-Departamento de Extensión Cultural; 1956.46p.

7. Cassells ES. A test concerning artificial cranial deformation and status from the Grasshopper site, East-Central Arizona. J Ariz Archaeolo and Historic Socie, Inc. Arizona State Museum. The University of Arizona. Tucson. En The Kiv 1972;37(2):84-92.

8. Munizaga JR. Deformación craneana intencional en América. Rev Chil Antropol. 1987;6:113147.

9. Brain Rt. The decorated body. New York. USA: Harper \& Row, Publishers, Inc.; 1979.192p.

10. Blakely R, Mathews DS. Demographic model of the Etowa village population. Bulletin of the Georgia Academy of Science. 1975;33:166-179. 
11. Garret J. Status, the warriors class, and artificial cranial deformation. En: The King site: Continuity and con tact in Sixteenth Century, Georgia. Robert Blakely (Ed.). USA: University of Georgia Press. 1988; 35-46.

12. Schuwarz F. El enigma precolombino. Barcelona: Ediciones Martinez Roca: 1988. 270p.

13. Imbelloni J. Estudios de la morfología exacta, Parte III: Deformaciones Intencionales del Cráneo en Sur América. Revista del Museo de la Plata 1924-1925;28:329-407.

14. Imbelloni J. "Intorno ai crani incredibili degli indiani Natchez". Atti del XXII Congreso Internationale degli Americanistli, Roma 1926-1928; volumen I, 391-406 p.

15. Imbelloni J. Los pueblos deformadores de los Andes. La deformación intencional de la cabeza como arte y como elemento diagnóstico de las culturas. Anales del Museo Nacional de Historia Natural Bemardino Rivadavia, t. XXXVII, Publicación No 75. Buenos Aires, 1933.p.

16. Kauffmann DF. Manual de arqueología peruana. Lima: ediciones Peisa. 1973.336p.

17. Dembo A, Imbelloni J. Deformaciones intencionales del cuerpo humano de carácter étnico. J. Anesi, Buenos Aires 1938: 249-277.

18. García HF, Murphy EG. Índice craneano en grupos étnicos precolombinos del Norte de Chile con deformación craneal artificial. Int. J. Morphol. 2009;27(2):587-594.

19. Weiss P. Osteología Cultural, 2da. Parte: Tipología de las deformaciones cefálicas - estudio cultural de los tipos cefálicos y de algunas enfermedades óseas. Lima: Universidad Nacional Mayor de San Marcos: 1960. 51-82p.

20. Weiss P. Las deformaciones cefálicas intencionales como factores de la arqueologia. XXXIX Congreso Internacional de Americanistas, Lima, Perú 1972; tomo 1, pp. 165-1 80.

21. Comas CJ. Datos para la historia de la deformación craneal en México. Historia Mexicana, México 1960;9: 509-520.

22. Sperber GH. Craniofacial Embriology. 4th edition. Wright, 1989.246p.

23. Bassett CA. A biological approach to craneofacial morphogenesis. Acta Morphol. Neerl. Scand 1972;10:71.

24. Arensburg B, Kaffe I, Littner MM. The anterior buccal mandibular depressions: ontogeny and phylogeny. American Journal of Physical Anthropology 1989;78:431-437.

25. Rosas A. Ontogenia y filogenia de la mandíbula en la evolución de los homínidos. Aplicación de un modelo de morfogénesis a las mandíbulas fósiles humanas de Atapuerca. [Tesis de Licenciatura. Biología Animal]. Madrid, Universidad Complutense de Madrid. 1992.

26. Bastir M, Rosas A. Facial heights: evolutionary relevance of postnatal ontogeny for facial orientation and skull morphology in humans and chimpanzees. Journal of Human Evolution 2004a; 47: 359-381.

27. Moss ML. The primary Role of Functional Matrices in Facial Growth Am. J. Orthod 1989;55:566-577.
28. Enlow DH. Crecimiento maxillofacial. 2a ed. México D.F.: Nueva editorial interamericana. $1984.508 \mathrm{p}$.

29. Hoyte DA. The modes of growth of the neurocranium: the growth of the sphenoid bone in animals. In: Cranio-facial Growth in Man. Ed. By R.E. Moyers and W. M. Krogman. Oxford, Pergamon Press, 1971p.

30. Limborgh, $L$ van. A new view on the control of the morphogenesis of the skull. Acta Morphol. Neerl. Scand 1970; 8:143.

31. Limborgh $\mathrm{L}$ van. The role of genetic and local environmerntal factors in the control of postnatal craniofacial morphogenesis. Acta Morphol. Neerl. Scand 1972;10:37.

32. Proffit WR. Ortodoncia teoría y práctica. 3a. ed. Madrid: Harcourt, 2001.666p.

33. Retzlaff EW, Michael D, Roppel $\mathrm{R}$, Mitchell F. The structures of cranial bone sutures. J Am Osteopth Assc 1976;75(6):607-608.

34. Meike MC. Craniofacial Development, Growth and Evolution. 1st edition. Bateson Publishing, Bressingham, Norfolk, England, 2002.363p.

35. Meindl RS, Lovejoy CO. Ectocranial suture closure: a revised method for the determination of skeletal age at death based on the lateral-anterior sutures. Am J Phys Anthropol 1985 Sep; 68(1):5766.

36. Lapunzina P, Aiello H. Manual de Antropometría normal y patológica - Fetal, neonatal, niños y adultos. Barcelona España: Masson, 2002.534p. 